BULL. AUSTRAL. MATH. SOC.

VOL. 12 (1975), 479.

\title{
A geometric theory of wild arcs
}

\section{Geoffrey John Hemion}

A topological embedding of a $1-c e l l$ in the 3-sphere is called an arc. Arcs which are wild at a single point have been the subject of a number of studies over the past twenty-five years. They have proven to be interesting in their own right as well as being sources for examples of topological embeddings in 3-manifolds.

This thesis is devoted to a characterization of arcs which are wild at a single point, at which they have finite penetration index, in terms of sequences of tame sets. In order to do this the notion of a "prime" arc is defined and a unique prime decomposition theorem is proved. The prime arcs have the property that "levels" of "echelon neighbourhoods", which are 3-cells surrounding the wild point, can be defined. The characterization involves studying these levels.

If the penetration index is three the characterization becomes particularly easy and Chapter 6 is devoted to a description of arcs of penetration index three.

Received 8 January 1975. Thesis submitted to the Australian National University, October 1974. Degree approved, March 1975. Supervisor: Dr J.M. McPherson. 